Rev. Latinoam. Psicopat. Fund., São Paulo, 18(1), 47-61, mar. 2015

http://dx.doi.org/10.1590/1415-4714.2015v18n1p47.4

\title{
Figure culturelle de la puberté: l'adolescent et le sport
}

\author{
Cristina Lindenmeyer*1
}

S'il existe un corps qui interroge l'envers de la culture et met à l'épreuve la dynamique pulsionnelle c'est bien le corps pubère. Lieux de transformation et de métamorphoses importantes, le corps pubère bouscule les certitudes infantiles et réactive le complexe d'Edipe, venant de ce fait interroger le social. Comment l'adolescent s'y prend-il pour supporter cette traversée dans la «tempête»? Le bouleversement pulsionnel met-il en échec la symbolisation de l'adolescent? Quels sont les fantasmes réactivés par la transformation pubertaire? Comment la société prend-elle en compte les modalités subjectives présentes dans cette période? Dans ce texte l'auteur propose une réflexion sur la pratique du sport engagée comme solution de compromis pour négocier avec la dynamique pulsionnelle bouleversée par le réel du corps pubère. Une figure clinique vient illustrer le propos.

Mots clés: Adolescence, corps pubère, sport, complexe d'Edipe

${ }^{* 1}$ Université Paris 7 - Diderot (Paris, France). 
Nous savons depuis Freud (1929/2010) que refouler les pulsions au profit du mouvement civilisateur demeure la tâche imposée au petit enfant. Puisque les pulsions refoulées ne disparaissent pas, elles doivent trouver des solutions de compromis pour négocier avec un conflit permanent et douloureux, qui constitue de ce fait le terreau de la névrose. La culture prend racine dans ce terrain conflictuel et des formations substitutives ayant l'aspect d'illusions viennent combler les désirs infantiles refoulés. Le sport est une de ces solutions sublimatoires apportée par notre culture.

Cependant s'il existe un corps qui interroge l'envers de la culture et la met à l'épreuve c'est bien le corps pubère. Lieux de transformation et de métamorphoses importantes, le corps pubère bouscule les certitudes infantiles et réactive le complexe d'Edipe, venant de ce fait interroger le social. Comment l'adolescent s'y prend t-il pour supporter cette traversée dans la «tempête»? Le bouleversement pulsionnel met-il en échec la symbolisation de l'adolescent? Quels sont les fantasmes réactivés par la transformation pubertaire? Comment la société prend-t-elle en compte les modalités subjectives présentes dans cette période?

Dans ce texte je m'appuie sur la notion de pulsion proposée à partir des textes freudiens. Je rends compte de l'expérience de puberté comme d'un temps de rupture entre le corps enfant et le corps adulte qui fait émerger l'intrication entre la psyché et le soma. Je prends appui, à titre d'illustration, sur le cas clinique de Célestine une jeune fille athlète de niveau olympique, qui, au moment de sa puberté et de part son contexte familial, va vivre ce moment comme un non retour, un temps de rupture chargé de fantasmes.

\section{Le corps pubère}

La puberté est un moment d'altération organique venant interroger les dynamiques pulsionnelles qui habitent l'adolescent. 


\section{ARTIGO}

Freud (1905/2006) avait bien perçu le malaise de l'adolescent lorsqu'il écrivait dans «Trois essais sur la sexualité infantile»:

Avec l'arrivée de la puberté interviennent les transformations qui doivent faire passer la vie sexuelle infantile à sa configuration normale définitive. La pulsion sexuelle était jusqu'ici principalement auto-érotique, elle trouve maintenant l'objet sexuel. Elle s'exerçait jusqu'ici à partir de pulsions et des zones érogènes isolées qui, indépendamment les unes des autres, cherchaient comme but sexuel unique un certain plaisir. (...) Comme le nouveau but sexuel assigne des fonctions très distinctes aux deux sexes, leur développement sexuel diverge désormais largement. (p. 145)

Ce passage ouvre la dimension organique du corps qui s'impose, le plaçant ainsi dans une étrangeté qui fait émerger des contenus jusqu'alors pris dans des fantasmes liés aux plaisirs auto-érotiques infantiles. Corps de mutations importantes qui bouscule les certitudes infantiles et réactivent le complexe d'Edipe, se présentant ainsi dans une «reconfiguration» nécessaire.

Des corps devenus trop grands dans une âme de tout petit; des voix qui muent, trahissant les changements internes; des corps malmenés, trop maigres ou trop gros, envahis par des mouvements incontrôlables, tous ces corps livrent le débordement incontrôlable du pulsionnel.

Comment le corps pubertaire à la merci de ces transformations réelles et des fantasmes qui l'accompagnent pourra-t-il continuer à être investi ainsi par le sujet qui n'est plus un enfant et pas encore un adulte? La poussée pubertaire impose à l'économie psychique une tyrannique nécessité de se défaire des voies anciennes de plaisir pour aller vers d'autres formes de satisfactions pulsionnelles.

\section{Corps pulsionnel, corps érotique}

Pourquoi le corps pubère est-il donc le siège de tels bouleversements?

En subvertissant l'idée d'un corps qui ne serait qu'anatomique Freud ne va pas seulement mettre en rapport le corps et la sexualité infantile, il va aller plus loin en précisant que c'est à partir du corps que tout prend naissance. On assiste alors à l'émergence de la psychanalyse et à l'idée qu'autour de l'anatomie corporelle se construit une vie fantasmatique intense. La vision de l'anatomie du corps devient une scène qui en cache une autre.

Bien qu'héritier de la clinique médicale, Freud s'en éloignera progressivement. En effet si nous pouvons envisager le corps à travers la vision du cadavre, un être mort comme objet d'étude pour la médecine, il en va tout autrement pour la psychanalyse. Pour Freud il ne peut se réduire à l'organique seul ni être compris 
dégagé de ses engagements d'avec l'organique. Le corps reste le lieu d'émergence de toute la dynamique pulsionnelle du sujet. Il est un lieu d'ancrage pulsionnel mais aussi un moyen pour le sujet d'arriver à satisfaire ses pulsions que se soit dans le plaisir ou le déplaisir.

Tout au long de l'œuvre freudienne le corps va ainsi traverser l'évolution de certains concepts fondamentaux pour la psychanalyse que ce soit la rencontre avec les manifestations somatiques chez les patientes hystériques où la référence plastique est attribuée au corps, ou que ce soit par le narcissisme lorsque le corps est pris comme objet d'amour. L'idée se prête à l'action d'une sexualité qui sera soutenue comme omni présente et par l'instauration de la pulsion. C'est leur présence dans le corps à corps avec l'autre qui en fera un lieu érogène siège d'une vie fantasmatique.

Avec le concept de pulsion, le lieu du corps est désigné comme source et comme moyen de satisfaction libidinale. Certaines parties corporelles utilisées par les pulsions permettent la mise en place de scènes fantasmatiques et imaginaires plus diverses.

Dans «Pulsions et destins des pulsions», Freud (1915/2009) reprend le point de vue exposé dans les «Trois essais sur la théorie sexuelle», et définit la pulsion comme une poussée dynamique qui a une source, un but et un objet, puis il en décrit les implications. J. Laplanche et J.-B. Pontalis dans Vocabulaire de la psychanalyse font observer que Freud ne confond pas instinct (Instinkt) et pulsion (Trieb) «l'instinct a un but préformé et adapté à un objet fixe alors que la pulsion est un processus dynamique comportant quatre éléments, source, poussée (constante), but et objet, et a au contraire un objet variable et contingent» $(1964$, p. 369). Ce dernier lui permet d'atteindre son but qui est de supprimer l'état de tension qui règne à la source. Pour Jean Laplanche, J.-B. Pontalis et Freud, le terme «source de la pulsion» est réservé au processus organique et physico-chimique à l'origine de l'excitation interne et est un moment somatique, non psychique «Par source de la poussée pulsionnelle, on entend le processus somatique qui est localisé dans un organisme ou une partie du corps et dont l'excitation est représentée dans la vie psychique par la pulsion » (1915/2009, p. 19). On peut alors comprendre le «moment somatique» (Assoun, 1997) comme celui de l'instinct et de la pulsion sous une forme «psychisée».

Dans la suite du texte «Au-delà du principe du plaisir», Freud introduit avec précaution, et en lui accordant un caractère spéculatif, la dernière théorie des pulsions, où s'opposent la pulsion de vie et la pulsion de mort. En même temps elles viennent en quelque sorte se rééquilibrer dans une «sorte de rythme-hésitation dans la vie des organismes ; un groupe avance impétueusement pour atteindre au plus tôt le but final, l'autre recule d'un bond, à un certain endroit de ce chemin, pour le parcourir une fois encore à partir d'un point déterminé et allonger ainsi la durée du chemin» $(1920 / 1996$, pp. 312-313). 


\section{ARTIGO}

Cette nouvelle conception dualiste est nécessaire pour pouvoir retenir l'idée de deux types de fonctionnement psychique avec, sur un pôle, un fonctionnement mental de liaison, et sur un autre pôle, un fonctionnement mental de dé liaison. L'appareil psychique va alors avoir pour tâche de lier les énergies libres. Si cette liaison ne s'établit pas, une perturbation du fonctionnement psychique qui ressemblerait à la névrose traumatique se déclenche. Le rétablissement du principe de plaisir dépend de la réussite de cette liaison. C'est ainsi que Jean Laplanche (1986) fidèle aux conceptions freudiennes caractérise les deux mécanismes «En d'autres termes, il est nécessaire de retenir une conception qui oppose deux types de fonctionnement mental, et nous retrouvons là l'opposition entre système lié et système non lié» (p. 90).

Avec la deuxième théorie des pulsions on commence à comprendre la place de l'autre dans le registre du corps. C'est par sa médiation que la pulsion va avoir un destin.

Rappelons ce qu'écrit Freud dans son texte «Le moi et le ça» à propos du moi corporel «Le moi est avant tout un moi corporel, il n'est pas seulement un être de surface, mais il est lui-même la projection d'une surface» (1923/1981, p. 238).

L'idée sous-jacente serait que le moi corporel soit un lieu d'accueil et d'impact provenant de l'autre. C'est précisément parce que Freud observe un lien étroit entre l'organisme et le milieu que le corps va être compris à partir de l'incidence de l'autre sur cet organisme, comme étant l'autre qui permettra la transformation des forces pulsionnelles. Mais de là émerge immédiatement la question sur la manière dont l'autre va accueillir cette décharge.

La pulsion de vie, ou Eros, cherche à intensifier la vie, à maintenir des liens de continuité. Ainsi elle correspondrait à ce qui a été développé dans le texte «Pour introduire le narcissisme», c'est-à-dire pouvoir s'aimer, grâce à celui qui, un jour a pu placer l'enfant du côté d'Eros. Déjà la pulsion de mort a des effets désorganisateurs sur les liaisons psychiques fondées sur ce registre narcissique.

André Green, dans un texte où il débat du concept métapsychologique de pulsion de mort, propose de distinguer deux fonctions qui caractérisent la pulsion de vie et la pulsion de mort. La fonction objectalisante est caractéristique des pulsions de vie et la fonction désobjectalisante caractéristique de la pulsion de mort. A. Green (1983) cible sa discussion sur la possibilité de se mettre en «relation à l'objet»: «Ceci justifie l'attention accordée aux théories de la relation d'objet, dont le tort est cependant de ne pas avoir clairement aperçu la fonction objectalisante pour s'être trop attachées à l'objet stricto sensu» (p. 55).

Cette fonction objectalisante de la pulsion de vie a pour effet de rendre possible l'établissement d'une relation à l'objet qui s'accomplit par un «investissement significatif» (p. 55) avec le paradoxe qu'il repose sur le sujet qui peut se prendre lui-même comme objet d'investissement. A l'opposé, la fonction 
désobjectalisante propre à la pulsion de mort, insiste sur la destruction ou sur le désinvestissement de la relation à l'objet. De là un autre paradoxe si l'on considère que le sujet peut se prendre comme objet de désinvestissement. Autrement dit, toute relation d'objet (interne) — ou ses substituts — est susceptible d'être attaquée à tout moment.

Revenons sur les soins prodigués à l'enfant. S'en occuper c'est lui transmettre des messages. Les soins investis par la personne qui les donne, le contient par l'affect vont constituer comme corps pulsionnel dans le champ sexuel. La façon dont la personne soignante répondra à ces forces pulsionnelles, permettra au corps de se constituer. Il existe ainsi une pénétration de l'autre qui reste initiale et qui fait que ce corps devient un «sujet-corps» (Birman, 1997). Ce «sujet-corps» est avant tout le résultat de cette espèce de " collage » entre d'un côté, les forces pulsionnelles et de l'autre côté leur effet sur le sujet. C'est donc le trajet pulsionnel qui passe par l'autre et qui donne à l'organisme sa possibilité de devenir un corps.

La notion de corps érogène introduite par Freud dans son texte "Trois essais sur la théorie sexuelle» dessine l'évolution du courant autoérotique qui se met en place à partir des modalités de réponse que l'adulte impose au nourrisson au moment de la satisfaction ou de l'insatisfaction de ses besoins. Le corps érogène s'organisera selon les premiers éprouvés sensoriels impliquant la présence ou l'absence de l'autre en ce qui concerne la satisfaction des besoins vitaux. C'est donc de la qualité de l'entourage ou de «l'autre qui porte soin» que dépendront les premiers investissements que l'enfant fera de son propre corps.

Si l'excitation naît en lien avec des fonctionnements organiques, elle s'organisera de façon libidinalisée à partir des premières sensations que procurent les soins.

Le corps érogène se met en place en deux temps: le premier est celui de l'investissement de la sexualité menant au narcissisme; le deuxième se joue à la puberté avec la sexualisation de l'organe génital et toute la force explosive introduite par la métamorphose pubertaire.

Le premier temps est fortement dépendant de l'environnement et de la façon dont l'enfant expérimente ce corps à corps avec l'autre. D. W. Winnicott (1957-1963/1970) qualifiera d'absolue la dépendance du nourrisson à l'égard de l'environnement. A cause de la non-intégration primaire du nourrisson, ce dernier passera par des moments d'"angoisse inimaginable" (p. 11) et d'annihilation, dont l'équivalent est l'angoisse de morcellement qui donne le sentiment de ne pas cesser de tomber et de se détacher de son propre corps. L'angoisse primaire retrouvée chez les patients schizophrènes, traduit vraiment l'importance essentielle des soins apportés au nourrisson. Sous la plume de D.W.Winnicott leur description est traduite par le terme holding, illusion d'une image interne «contenante» et protectrice.

J. Laplanche, quant à lui, a pris en compte la position anthropologique originelle qui caractérise l'être humain, à savoir, le fait qu'il arrive au monde au 


\section{ARTIGO}

bout de neuf mois, non fini, et dans la contrainte d'être pris en charge par un autre. Il développe alors sa «Théorie de la séduction généralisée» (1987). Selon lui, conjointement à cette situation anthropologique fondamentale, se trouve aussi la position dissymétrique dans laquelle est l'enfant vis à vis de l'adulte qui lui donne les soins nécessaires, position essentielle sur le plan de la sexualité, et vient inscrire à jamais la présence du sexuel vers l'instauration du psychique. C'est à partir de cette situation entre un adulte qui a un inconscient marqué par le sexuel, constitué par sa propre sexualité infantile, et un enfant qui n'a pas encore les «montages sexuels génétiques» suffisants pour comprendre et élaborer ce sexuel adulte, que la rencontre s'instaure. Dans cette situation de «séduction originaire», il ne s'agit pas seulement d'un adulte qui accompagne et qui donne les soins mais de l'importance d'une introduction d'éléments inconscients qui se font malgré lui. Le geste de nourrir, par exemple, ne se réduit pas seulement à apporter de la nourriture. Dans ce geste il y a aussi un sein qui porte en lui une histoire libidinale. Ainsi aux moments des soins quelque chose s'implante et produit de l'excitation. Ce lien originaire du tout début de la vie reste donc contaminé par des messages chargés du sexuel adulte, ce que J. Laplanche désignera par le terme, "messages énigmatiques" (1987); énigmatiques, puisque sexuels, énigmatiques, puisque l'enfant n'a pas encore accès à la traduction de ce qui lui arrive. Ce terme «messages énigmatiques» prend toute son importance, car avec lui un autre mouvement vient s'ajouter, celui de traduire ces messages pour les intégrer au psychisme en cours de constitution. Cependant, du fait même de la situation dissymétrique, la traduction ne pourrait jamais être complète, laissant ainsi des restes en devenir.

Dans un premier temps les messages sont implantés, sans être pour autant traduits. C'est dans l'après coups et tout au long de l'évolution de l'enfant, que ces messages reviendront de l'intérieur pour trouver une traduction possible. Puis, à la puberté, tout le vécu fantasmatique infantile se réactive, ce qui fera dire à Freud que l'effet d'effraction d'un souvenir est rendu possible par la maturation physique, caractéristique propre à la puberté. Autrement dit, la nature du souvenir allié à l'émergence de la métamorphose organique du corps en voie de génitalisation ouvre cet effet explosif par la réactivation de certains vécus infantiles endormis sous l'effet du refoulement.

\section{Le sport, une figure culturelle}

Reconnu comme un «plaisir physique» débuté depuis longtemps dans l'histoire de la civilisation, le sport est considéré comme un moyen de promouvoir la santé, l'éducation et l'épanouissement personnel. 
Quiconque a déjà expérimenté les sensations agréables ressenties à la suite d'exercices physiques, se souvient du sentiment de satisfaction et de liberté tels que le décrivent à leur tour certains adolescents.

En effet, les effets bénéfiques, et nous pouvons aussi dire plaisants, de la pratique sportive sur la santé sont globalement connus. Elle fait travailler les muscles, brûle les calories et facilite l'évacuation de la tension nerveuse accumulée dans la journée, permettant la décharge momentanée de l'excitation vers son apaisement.

Chez l'adolescent, elle permet la maîtrise d'un corps mis à l'épreuve. Les sensations douloureuses qui l'accompagnent provoquent une nouvelle appréhension d'un corps devenu presque étranger et qui part à la redécouverte de ses limites. Le sport de haut niveau qui emmène plus loin que la simple pratique sportive, incite au dépassement de ses propres limites et s'inscrit dans une quête qui oscille entre l'auto estime de soi et l'envie de pouvoir. Vouloir gagner c'est prendre la place de l'autre - père, mère, frère, sœur...-. Mais qu'elle soit compétitive ou classique, l'activité sportive fait ressurgir les angoisses infantiles liées aux affrontements anciens, comme entre frères par exemple, et ce, de façon tellement violentes qu'elles frisent parfois l'insupportable.

Cependant, pour d'autres, elle est un moyen de plaisir jusqu'à devenir parfois un moyen de fuite face à un état dépressif latent. Un auteur comme C. Carrier (1996) a souligné l'utilisation du sport chez le sportif de haut niveau comme étant un mécanisme d'évitement des conflits là où le narcissisme et les échecs identificatoires sont au premier plan.

Dans notre cas clinique qui suit, Célestine s'y est confrontée jusqu'au moment où ce foyer de plaisir, investi comme «solution de vie» s'est transformé en support d'angoisse.

\section{Célestine et la pratique du sport}

Célestine est une jeune adolescente qui vient consulter pour un état «dépressif» qu'elle fait remonter à ses 12 ans. A cette époque, elle désinvestit de plus en plus certains secteurs de sa vie. Ses notes baissent sensiblement à l'école, les compétitions auxquelles elle participe la motivent moins, elle se blesse régulièrement lors des entraînements et elle reste souvent enfermée dans sa chambre en n'ayant envie de voir personne. Quand je la rencontre pour la première fois, elle explique qu'elle ne comprend pas ce qui lui arrive et que même la pratique du sport qui depuis toute petite était sa «raison de vivre» n'a plus de sens. Elle ne sait plus où elle en est et ne sait pas si elle va continuer ses activités sportives. 


\section{ARTIGO}

Célestine évoque les plaisirs réveillés par la sensation d'efforts qui lui donnent l'envie de la conquête alliée au sentiment de toute puissance que tout sportif éprouve lorsqu'il est gagnant. On est loin de la simple pratique d'un exercice quotidien. A ce niveau, le sport dépasse le divertissement; il mobilise les fantasmes pour tenter de toucher le spectateur et frapper l'adversaire. C'est ainsi qu'elle en parle comme d'une libération jouissive faisant surgir à mes yeux une autre scène. Elle confirme ce que Freud affirme: «il remplace la jouissance sexuelle par le plaisir du mouvement et ramène l'activité sexuelle à ses composantes auto-érotiques» (1905/2006, p. 104).

Tout au long des séances et grâce à ses associations, deux éléments prendront le devant de la scène: la séparation de ses parents et les modifications vécues dans son corps pubère. Ces deux éléments paraissent fonctionner comme des bombes faisant émerger des conflits jusque là inavouables. Selon l'apport psychanalytique, l'émergence de la puberté fonctionne comme un objet carrefour qui libère les mouvements œdipiens, suivis de vécus angoissant lies aux expériences corporelles d'avec le premier objet d'investissement (Gutton, 1991). Elle fait parfois régresser le sujet vers des vécus fantasmatiques proches de relations «incestuelles» (Racamier, 1995) dans un risque psychotisant. Dans cette frontière fragile, souligne F. Richard (2013), il s'agit de préserver l'adolescent des risques de décompensations majeures: «Commencer par analyser le complexe d'Oedipe pour seulement ensuite élucider l'archaïque préserve la relation à la réalité là où l'adolescent est menacé de basculer dans un univers où l'imaginaire envahit tout» (p. 337).

Chez Célestine, le rôle déterminant du fantasme œdipien dans le sport apparaitra assez vite et sera suivi d'éléments plus narcissiques. Fille unique d'un couple très engagé dans son éducation et ses entraînements, Célestine se retrouve l'objet de réparation d'une mère fortement affaiblie après une séparation conjugale et d'un père donc elle éveille l'admiration. Le moment de la séparation de ses parents voit se réactiver un conflit œedipien à une période où naissent les transformations corporelles pubertaires, ce qui fera dire comme P. Jeammet (1991), que c'est un «véritable passage à l'acte de la nature qui offre au préadolescent un corps apte à réaliser ses fantasmes» (p. 142). Coincée fantasmatiquement dans une représentation maternelle omniprésente, mais faible et seule et auprès d'un père adoré mais lointain, Célestine refoule ses affects ambivalents, ce qui fait naître en retour un état dépressif. En effet, comment continuer à éveiller l'admiration d'un père qui la place en position de rivalité vis à vis de la mère, sans aggraver les difficultés maternelles?

Dans un de ses derniers textes sur la «Sexualité féminine» Freud (1931/1969) souligne les mouvements œdipiens en lien avec les conflits préœdipiens chez la fille. Selon lui deux difficultés majeures habitent le complexe œdipien féminin et interrogent. Comment se débarrasser de la fixation à la figure maternelle, pour 
ensuite investir le père? Dans le corps, comment s'opère le changement d'organe du clitoris au vagin?

Il développe le rapport homosexuel primaire complexe à la mère, refoulé d'une façon particulièrement implacable et le point de fixation de l'hystérie. C'est parce que le détachement est à la fois absolument nécessaire et absolument insupportable que les choses se complexifient pour la fille. Dans un autre texte un an plus tard, Freud dira «L'acte de se détourner de la mère se produit sous le signe de l'hostilité, la liaison à la mère débouche dans la haine. Une telle haine peut devenir très frappante et se maintenir toute la vie; elle peut plus tard être soigneusement surcompensée; en règle générale, une partie d'elle est surmontée, une autre partie subsiste» (1932/1995, p. 205.) Au moment de la puberté, les transformations corporelles produisent un afflux excessif de la poussée libidinale, où re émergent sur la scène les fantasmes jusqu'alors refoulés. Le complexe d'CEdipe fait son retour et l'angoisse de castration qui s'impose avec les transformations corporelles, réapparaît. Comment ces angoisses actuelles vont-elles se dégager des angoisses d'intrusion prégénitales? C'est sur ce terrain qu'on trouve le paradoxe qui caractérise les fantasmes de Célestine. Comment se séparer de l'objet maternel, sans que cette séparation n'entraîne sa perte ou sa destruction? La difficulté vient d'une confrontation à ce noyau originaire qui draine des fantasmes hostiles avec risque 56 d'un état dépressif. S'en protéger, c'est mettre en place une action défensive sur le mode d'un refus d'exercer le sport et d'en geler ainsi son développement.

Ce mouvement chez Célestine rejoint les propositions d'un attachement sans fin entre mère et fille développées par M. Torok. En explorant les mouvements œdipiens en lien avec les conflits prégénitaux et potentiellement liés à la relation mère fille de la phase anale, Maria Torok dans son texte «La signification de l'envie du pénis chez la femme» (1964) discute la thèse freudienne sur le thème de la sexualité chez la fille. D’après Freud (1931/1969) l'envie du pénis apparaît comme la source de la sexualité féminine. Il inscrit le féminin dans la déception de ne pas appartenir à l'autre sexe «La différence qui réside dans cette part du développement sexuel de l'homme et de la femme est une conséquence naturelle de la différenciation des organes génitaux et de la situation psychiques qui s'y rattache; elle correspond à la différence entre castration accomplie et simple menace de castration» (1925/1973, p. 130).

Freud, prisonnier de son époque, déterminera les différences entre Oedipe masculin et féminin en se basant sur la différence anatomique poussant la fille à se forger un Surmoi lâche et faible. Maria Torok sort de cette dimension idéalisée et problématique. D'après elle la source de la sexualité trouve son origine dans le conflit psychique éprouvé par la petite fille dans sa relation précoce à la mère. Le conflit atteint son paroxysme lors de la phase de contrôle et de maîtrise sphinctérienne anale. A cette phase elle pense que la petite fille a déjà l'expérience 


\section{ARTIGO}

de l'exploration de son propre sexe lors de sa vision de la différence des sexes. Cependant il se serait passé quelque chose qui l'incite à abandonner cette partie si importante de son corps au profit d'un objet censé être inaccessible et inexistant chez elle. Ce quelque chose, selon Maria Torok, serait dû à la force du refoulement au profit de sa relation avec la figure maternelle. Le véritable enjeu qui abrite l'envie du pénis serait le refoulement inconscient de la haine adressée à l'imago maternelle. Pour ne pas perdre son amour, la petite fille se soumet aux exigences de la propreté imposée par le rôle éducatif de celle-ci. Elle renonce donc aux satisfactions érotiques anales. Les pulsions agressives consécutives au renoncement seront refoulées afin de protéger son rapport à la mère. Le refoulement de ses propres désirs maintient le serment de fidélité à cette dernière «... La maîtrise exercée par la Mère, dira M. Torok, ne peut être interprétée par l'enfant que comme une manifestation de son intérêt à l'égard de la possession des matières et cela dès leur intérieur du corps» (1964/1991, p. 164).

Avec le contrôle sphinctérien et l'apprentissage de la propreté, c'est tout l'intérieur du corps qui tombe sous le contrôle maternel. Tout se passe comme si la mère s'accaparait l'intérieur du corps de sa fille y compris ses organes internes féminins jusqu'à son vagin. Toujours selon Maria Torok, la petite fille pour se conforter avec les interdits, refoule l'existence de son vagin et inhibe toute sensation de plaisir. Dans les situations où la mère est perçue comme fragile et défaillante, l'enfant ne peut pas se construire un imago maternel autonome. A la place se crée une imago maternel vide, insatisfait et jalouse. Seule la maîtrise de l'enfant lui procure une satisfaction.

Cette théorie s'ajoute à celle de Ferenczi (1932/1983) quand il nous dit que le sujet peut se positionner comme protecteur, même au détriment de lui-même si cela lui assure la répression de son agressivité et la garantie de l'amour de l'objet investi. Dans cette perspective et à partir des situations cliniques Maria Torok constate que des femmes en contact avec une sensation de plaisir, tombent dans une forte culpabilité, puisque l'autonomie que procure l'accès au plaisir signifie pour la petite fille une dépossession sadique de la mère. Le Surmoi ainsi sollicité ne lâchera pas facilement.

Toute sensation de plaisir fonctionne comme un acte masturbatoire et est vécue comme le moyen par lequel la petite fille peut se détacher de sa mère.

Quand les conflits prégénitaux perdurent, la séparation d'avec la mère devient impossible à supporter pour l'enfant. Tout acte de plaisir est vécu comme destructeur pour la mère.

En m'appuyant sur ses hypothèses, je pense que les fantasmes soutenus par ces significations terrorisantes ont réapparu lors de la séparation des parents et des modifications vécues dans son corps pubère. Les sentiments archaïques restés secrets pendant l'enfance, ont alors pris une dimension si terrifiante que Célestine se donnera en sacrifice pour prendre ainsi la place de l'objet haï et introjecté à l'intérieur de soi. 


\section{Pour conclure}

La compréhension du rôle du corps chez le pubère m'a semblé indispensable pour saisir la problématique de l'utilisation du sport pour les adolescents. En effet, si pour certains adolescents la pratique sportive peut s'inscrire comme un moyen culturel qui traite l'excitation pulsionnelle, pour d'autres, cette opération se trouve bloquée. C'est le cas de Célestine, où une problématique œdipienne et narcissique est venue s'immiscer et a marqué un point d'arrêt à son activité sportive.

\section{References}

Assoun, P.L. (1997). Corps et symptôme. Clinique du corps (vol. I). Paris: Anthropos/Economica, 1997.

Birman, J. (1997). Le corps et l'affect en psychanalyse. Une lecture critique du dis cours freudien. Che vuoi?, 7, 13-26.

Carrier, C. (1996). Le «musculaire», produit du travail de l'adolescent sportif de haut niveau, Adolescence, 14(2), 153-167.

Ferenczi, S. (1983). Confusion de langue entre les adultes et l'enfant. In Oeuvres complètes (t. IV). Paris: Payot (Travail original publié dans 1932).

Freud, S. (1969). Sur la sexualité féminine. In La vie sexuelle. Paris: PUF. (Travail original publié dans 1931).

Freud, S. (1973). Quelques conséquences psychiques de la différence anatomique entre les sexes. In La vie sexuelle. Paris: PUF. (Travail original publié dans 1925).

Freud, S. (1981). Le moi et le ca. In Essais de psychanalyse. Paris: Payot. (Travail original publié dans 1923).

Freud, S. (1995). La féminité. In Euvres Complètes (t. XIX, p. 205). Paris: PUF. (Travail original publié dans 1932).

Freud, S. (1996). Au-delá du principe de plaisir. In Euvres Complètes (t. XV). Paris: PUF. (Travail original publié dans 1920).

Freud, S. (2006). Les reconfigurations de la puberté. In Trois essais sur la théorie sexuelle, Oeuvres complètes (t. VI). Paris: PUF. (Travail original publié dans 1905).

Freud, S. (2009). Pulsions et destins des pulsions. In Métapsychologie. Paris: Gallimard. (Travail original publié dans 1915).

Freud, S. (2010). Malaise dans la civilisation. Paris: Payot. coll. «Petite Bibliothèque Payot». (Travail original publié dans 1929).

Green, A. (1983). Narcissisme de vie, narcissisme de mort. Paris: Minuit.

Gutton, P. (1991). Le pubertaire. Paris: PUF. 


\section{ARTIGO}

Jeammet, P. (1991). Les enjeux des identifications à l'adolescence. Journal de la psychanalyse de l'enfance, 10, 140-163.

Laplanche, J., \& Pontalis, J.-B. (1964). Vocabulaire de la psychanalyse. Paris: PUF, p. 369.

Laplanche, J. et al. (1986). La pulsion de mort dans la théorie de la pulsion sexuelle. In La pulsion de mort. Paris: PUF, p. 90.

Laplanche, J. (1987). Nouveaux fondements pour la psychanalyse. Paris: PUF.

Lindenmeyer, C. (2012). Qual é o estatuto do corpo na psicanálise? Tempo Psicanalítico, 44(2), 341-359.

Racamier, P.C. (1995). L'inceste et l'incestuel. Paris: Ed. du collège de psychanalyse familiale et groupale.

Richard, F. (2013). L'analyse après-coup de l'adolescent dans les cures d'adultes. Revue française de psychanalyse, LXXVII(2) 331-347.

Torok, M. (1991). La signification de l'envie du pénis chez la femme. In La sexualité féminine. Sous la dir. J. Chasseguet-Smirgel. Paris: Payot. (Travail original publié dans 1964).

Winnicott, D.W. (1970). Intégration du moi au cours du développement de l'enfant. In Processus de maturation chez l'enfant (1957-1963). Paris: Payot.

\section{Résumés}

(Figura cultural da puberdade: o adolescente e o esporte)

Se existe um corpo que afronta o social e coloca à prova a dinâmica pulsional, este corpo é o pubertário. Lugar de transformações e de metamorfoses importantes, o corpo pubertário varre as certezas infantis e reativa o complexo de Édipo. Como o adolescente suporta essa travessia na "tempestade"? A reconfiguração da dinâmica pulsional colocada à prova abala a capacidade de simbolização do adolescente? Quais os fantasmas que serão reativados pela transformação pubertária? Como a sociedade aborda as modalidades subjetivas presentes nesse periodo?

Neste texto, a autora propõe uma elaboração sobre a prática do esporte como solução de compromisso para negociar com a dinâmica pulsional perturbada pelo real do corpo pubertário. Uma figura clínica ilustra essa proposição.

Palavras-chave: Adolescência, corpo pubertário, esporte, complexo de Édipo, narcisismo

(Cultural figure of puberty: the adolescent and sports)

The pubescent body questions the backside of culture and puts to test pulsional dynamism. A place of transformation and metamorphosis, the pubescent body overthrows infantile certainties and reactivates the Oedipus complex, thus questioning social concepts. How does the adolescent crosses that "storm"? Does that pulsional upheaval make adolescent symbolization fail? What fantasies are reactivated by 
pubescent transformation? How does society take into account the subjective factors that come up during that period? In this context, the author suggests the practice of sports as a compromise to negotiate pulsional dynamism disturbed by the reality of the pubescent body. A clinical case illustrates that proposal.

Keywords: Adolescence, pubescent body, sports, Oedipus complex

(Figura cultural de la pubertad: el adolescente y el deporte)

Si existe un cuerpo que cuestiona lo social y que pone a prueba la dinámica pulsional, este es el cuerpo pubertario. Lugar de transformaciones y de metamorfosis importantes, el cuerpo pubertario analiza las afirmaciones infantiles y reactiva el complejo de Edipo. ¿Cómo hace el adolescente para soportar esta travesía en la “tormenta”? ¿La reconfiguración de la dinámica pulsional puesta a prueba debilita la capacidad de simbolización del adolescente? ¿Cuáles serán los fantasmas reactivados por la transformación pubertaria? ¿Cómo aborda la sociedad las modalidades subjetivas presentes en este periodo? En este texto, la autora propone una reflexión acerca de la práctica deportiva como solución de compromiso para negociar con la dinámica pulsional trastornada por la realidad del cuerpo pubertario. Un caso clínico ilustra la tesis.

Palabras claves: Adolescencia, cuerpo pubertario, deporte, complejo de Edipo

(Kulturfigur der Pubertät: der Adoleszent und Sport)

Wenn es einen Körper gibt, der die Schattenseiten der Kultur hinterfragt und die Triebdynamik auf die Probe stellt, so ist es sicherlich der pubertierende Körper. Der menschliche Körper, Gegenstand äußerlicher und innerlicher Veränderungen, zerrüttet die Sicherheit der Kindheit, lässt den Ödipuskomplex wieder aufleben und führt dazu, dass der Jugendliche die bestehende Gesellschaftsordnung hinterfragt. Wie verhält sich der Jugendliche, um diesen „Sturm“ zu überstehen? Sabotiert die triebliche Veränderung die Symbolisierung des Heranwachsenden? Welche Vorstellungen leben wieder auf, verursacht durch die pubertären Veränderungen? Wie berücksichtigt die Gesellschaft das subjektive Verhalten dieses Lebensabschnittes?

Dieser Text lädt anhand einer Fallbeschreibung zum Nachdenken ein, das Praktizieren einer körperlichen Aktivität (Sport) als einen möglichen Kompromiss anzusehen, um mit der Triebdynamik, die vom pubertierenden Körper umgewälzt wird, zu verhandeln.

Schlüsselwörter: Adoleszenz, pubertierender Körper, Sport, Ödipuskomplex 


\section{ARTIGO}

Citação/Citation: Lindenmeyer, C. (2015, março). Figure culturelle de la puberté: l'adolescent et le sport. Revista Latinoamericana de Psicopatologia Fundamental, 18(1), 47-61.

Editores do artigo/Editors: Manoel Tosta Berlinck e Sonia Leite

Recebido/Received: 30.11.2013/ 11.30.2014 Aceito/Accepted: 15.3.2014 / 3.15.2014

Copyright: (C) 2009 Associação Universitária de Pesquisa em Psicopatologia Fundamental/ University Association for Research in Fundamental Psychopathology. Este é um artigo de livre acesso, que permite uso irrestrito, distribuição e reprodução em qualquer meio, desde que o autor e a fonte sejam citados / This is an open-access article, which permits unrestricted use, distribution, and reproduction in any medium, provided the original authors and sources are credited.

Financiamento/Funding: A autora declara não ter sido financiada ou apoiada / The author has no support or funding to report.

Conflito de interesses/Conflict of interest: A autora declara que não há conflito de interesses / The author has no conflict of interest to declare.

\section{Cristina Lindenmeyer}

Psychanalyste; Maître de conférences à l'UFR-EP, Université Paris 7 - Diderot (Paris, France); Membre du CRPMS - UFR-EP à l'Université Paris 7 - Diderot.

44, rue de Sévigné

75003 Paris, Françe

e-mail: cristina.lindenmeyer@wanadoo.fr 